\title{
Prognostic implication of PD-L1 expression and associated tumor infiltrating lymphocytes in metastatic breast cancer
}

\author{
Mohamed M Darwish'1 , Atef Y Riad ${ }^{1}$, Dina A Salem ${ }^{1}$, Ahmad E Essa ${ }^{1}$, Marwa M Shakweer $^{2 *}$, Diaa Eldin M. Sherif \\ ${ }^{1}$ Department of Clinical oncology and nuclear medicine, Faculty of Medicine, Ain Shams University, Cairo, Egypt \\ ${ }^{2}$ Department of Pathology, Faculty of Medicine, Ain Shams University, Cairo, Egypt
}

\author{
*Correspondence to \\ Marwa M Shakweer, Email: \\ shakweer_13@med.asu.edu.eg
}

Received 22 Apr. 2021

Accepted 27 May 202

Published online 30 July 2021

Keywords: Metastatic breas neoplasm, PD-L1, Tumo infiltrating lymphocytes

\begin{abstract}
Introduction: One of the most important regulators of immune response is the programmed death receptor 1 (PD-1) and its interaction with its ligand (PD-L1), which negatively influences the immune response.

Objectives: This study aims to clarify PD-L1 expression levels and the associated tumor infiltrating lymphocytes (TILs) in patients with metastatic breast cancer, and to assess their influence on the prognosis of these patients and the association with clinico-pathologic criteria.

Patients and Methods: PD-L1 expression was analyzed using immunohistochemistry (IHC) while TILs count was assessed by histopathological examination of the hematoxylin and eosin (H\&E) stained full tumor sections from 50 patients diagnosed with stage IV breast cancer at Ain Shams University hospital, Cairo, Egypt.

Results: PD-L1 expression was demonstrated on TILs in 21 of 50 specimens, and on tumor cells in 13 of 50 specimens. Triple negative breast cancer (TNBC) and ER-/Her2+ subtypes were significantly associated with TIL infiltration and PD-L1 expression (on TILs and tumor cells). High TIL infiltration was significantly associated with worse overall survival (OS) and progression free survival (PFS) $(P=0.0238$ [HR 4.7, 95\% Cl: 1.22-18.11] and $P=0.0262[\mathrm{HR} 3.1,95 \% \mathrm{Cl}: 1.14-8.59]$ respectively). No correlation was found between PD-L1 expression (on tumor or TILs) and the survival of the patients (OS nor PFS).

Conclusion: High TIL count infiltrating the breast tumor is associated with worse OS and PFS in patients with metastatic breast cancer. High PD-L1 expression correlated with high counts of TIL levels around the tumor These findings have major clinical implications in using immune-checkpoint inhibitors in treating breast cancer patients.
\end{abstract}

\section{Introduction}

Globally, breast cancer is the second most frequently diagnosed malignancy just behind lung cancer, accounting for over two million cases each year, and the fourth cancer causing death worldwide; the leading cause of cancer death though in females, with around 600000 deaths each year (1).

Cancer immunotherapy through inhibition of negative immune regulators or checkpoints, represents a major progress in cancer therapy. Of the immune checkpoints to be targeted is the programmed cell death protein 1 (PD-1) and its ligand (PD-L1). The measurement of these two biomarkers is used as a prognostic and predictive marker to the immunotherapy with checkpoint inhibitors (2).

Inhibitors of PD-1 and PD-L1 were shown to significantly improve patient outcomes in different types of immunogenic cancers such as non-small cell lung cancers, malignant

\begin{abstract}
Key point
The role of PDL1 expression in breast cancer is still under investigation for possible application of immunotherapy as a new treatment modality. Triple negative breast cancer (TNBC) and ER-/Her2+ subtypes were significantly associated with tumor infiltrating lymphocytes (TILs) infiltration and PDL1 expression (on TILs and tumor cells). High TIL infiltration was significantly associated with worse overall survival (OS) and progression free survival (PFS). High TIL counts and PD-L1 positivity (on both TILs and tumor cells) were most significantly associated with ER-/Her2+ and TNBC. Further studies with large sample size are recommended to find correlation between PD-L1 expression (on tumor or TILs) and the survival of the patients (OS nor PFS).
\end{abstract}

melanoma, renal cell carcinoma, and some immunogenic breast cancer subtypes (3-6).

Breast cancer is composed of heterogenous subtypes; each has its specific pathologic and genetic characters, as well as different

\footnotetext{
Copyright ( $) 2022$ The Author(s); Published by Nickan Research Institute. This is an open-access article distributed under the terms of the Creative Commons Attribution License (http://creativecommons.org/licenses/by/4.0), which permits unrestricted use, distribution, and reproduction in any medium, provided the original work is properly cited.
} 
prognostic significance. Of these subtypes, triple negative breast cancer (TNBC) is the most immunogenic type, as it is rich in tumor infiltrating lymphocytes (TILs) and PDL1 co-expression (7).

In a phase III trial, combining atezolizumab and nabpaclitaxel conferred a non-statistically significant overall survival (OS) benefit compared with nab-paclitaxel alone in unselected, patients with TNBC. Interestingly, the difference in OS was statistically and clinically significant in the PD-L1 positive subgroup of patients, highlighting the potential clinical importance of PD-L1 expression as a predictive biomarker in breast cancer $(8,9)$.

\section{Objectives}

We aimed to evaluate the prognostic value of PD-L1 expression and associated TILs in stage IV breast cancer patients, and correlate them with response evaluation, progression free survival (PFS) and OS.

\section{Patients and Methods}

\section{Patients}

Tumor samples were collected at Demerdash Pathology Lab from 50 female patients with pathologically proven stage IV breast cancer between 2017 and 2019 presented at the breast cancer unit in Department of Clinical Oncology, Ain Shams University hospital, Cairo, Egypt. The ruled in patients did not receive any treatment yet to their metastatic disease. Patients who received adjuvant or neoadjuvant systemic treatment were enrolled in the study. Patients were followed up to at least one year after starting their systemic treatment. Patients with associated systemic immunologic diseases and pregnant patients were excluded from the study.

\section{Scoring methods}

The percentage of stromal TILs is determined by the area occupied by mononuclear inflammatory cells over total intra-tumoral stromal area within the borders of the invasive tumor (including the invasive tumor front).

Immunohistochemistry (IHC) staining was conducted on the paraffin embedded tissue sections with a labelled streptavidin- biotin-peroxidase complex technique using a rabbit monoclonal antibody for PD-L1 (CD274 molecule) (catalogue number: cell signaling, 13684; dilution: 1/200). Antigens were retrieved by microwaving in citrate buffer for 20 minutes for PDL1. To verify antibody specificity; placental tissue was used as a positive control for PDL1.

Scoring of TIL was based on the recommended methodology of the International TILs Working Group (10). The density of stromal TILs is recorded as a continuous percentage and categorized for analyses as; none or focal TILs if $<5 \%$, low in between $5-50 \%$, and high if $>50 \%$ (10).

PD-L1 scoring was conducted according to a previous study by Sobral-Leite et al. Cells with membranous expression of PDL1 were considered positive, while positive cytoplasmic or nuclear staining were nonspecific. PDL1-positive tumor cells (PD-L1+ tumor-cells) exhibiting membrane staining over the entire tumor were recorded as a continuous variable $(0-100 \%)$ and categorized for analyses as: negative if $<1 \%$ and positive if $\geq 1 \%$. PD-L1positive immune cells scoring was based on the percentage of the area occupied by stromal TILs exhibiting PD-L1 staining over the total area occupied by stromal TILs (0-90\%; as continuous variable). It was categorized as; negative or focal if $<5 \%$, moderate if between $5-50 \%$, and high if $>50 \%$ (7).

\section{Statistical analysis}

Baseline characteristics of the patients and tumor pathological features are expressed as absolute values, mean \pm standard deviation and median (range) when appropriate. A chi-square test was used to measure the association between these parameters. Survival data were presented on Kaplan-Meier curves and suitable tests were used to compare survival data. Results will be shown as $P$ values. Statistical significance is defined as $P<0.05$. Value of $P<0.01$ is highly significant whereas the $P>0.05$ is nonsignificant.

\section{Results}

The clinical and pathologic criteria of the patients enrolled in our study is summarized in Table 1.

This study included 50 female patients with treatment naïve metastatic breast cancer, with mean age 45.8 years (standard deviation 11.1, range 25 to 75 years). From this analysis, 35 patients (70\%) were pre-menopausal, while 15 (30\%) were post-menopausal. Regarding the metastatic deposits, 14 patients (28\%) had only bone metastasis, while 20 patients $(40 \%)$ had only visceral deposits. 16 patients (32\%) had de novo metastatic disease (metastatic from the start), while 34 patients (68\%) were relapsed after adjuvant/neoadjuvant therapy. As regards the type of first line metastatic systemic therapy received, 29 patients (61.7\%) received chemotherapy, 13 patients (27.7\%) received endocrine therapy, while only five patients (10.6\%) received combined chemotherapy plus endocrine therapy. Among the 50 patients of the study, 35 patients (70\%) were of the luminal subtypes, 8 patients $(16 \%)$ were ER negative/Her2 positive, while 7 patients (14\%) were TNBC.

We searched for correlations between PD-L1 expression and TIL infiltration in tumor specimens for patients with metastatic breast cancer, and their relation to the clinicopathological features of the patients.

We observed a strong correlation between TILs and PDL1 expression (on tumor and surrounding TILs $[P=0.0011$ and $P<0.0001$ respectively; Table 2]).

TNBC and ER-/Her2+ subtypes were the most common subtypes significantly associated with presence of TIL 
Table 1. The clinicopathologic data of the patients

\begin{tabular}{|c|c|}
\hline Characteristic & Number $(\%)$ \\
\hline Age (years) & $45.8 \pm 11.1$ \\
\hline \multicolumn{2}{|l|}{ Menopausal status } \\
\hline Post & $15(30)$ \\
\hline Pre & $35(70)$ \\
\hline \multicolumn{2}{|l|}{ Co-morbidities } \\
\hline No & $31(62)$ \\
\hline Yes & $19(38)$ \\
\hline \multicolumn{2}{|l|}{ Tumor grade } \\
\hline 2 & $33(66)$ \\
\hline 3 & $11(22)$ \\
\hline Missing & $6(12)$ \\
\hline \multicolumn{2}{|l|}{ Tumor intrinsic subtypes } \\
\hline Her2 enriched & $8(16)$ \\
\hline luminal $\mathrm{A}$ & $17(34)$ \\
\hline luminal B & $12(24)$ \\
\hline luminal B Her2 enriched & $6(12)$ \\
\hline TNBC & $7(14)$ \\
\hline \multicolumn{2}{|l|}{ De novo metastatic disease } \\
\hline No & $34(68)$ \\
\hline Yes & $16(32)$ \\
\hline \multicolumn{2}{|l|}{ Site of metastases } \\
\hline Viscera only & $18(36)$ \\
\hline Bone only & $15(30)$ \\
\hline Viscera and bone & $17(34)$ \\
\hline \multicolumn{2}{|l|}{ Burden of disease } \\
\hline Multiple & $45(90)$ \\
\hline Oligo & $5(10)$ \\
\hline \multicolumn{2}{|c|}{ Type of first line systemic treatment } \\
\hline $\mathrm{CTH}$ & $29(62)$ \\
\hline $\mathrm{CTH} /$ Endocrine & $5(10)$ \\
\hline Endocrine & $13(28)$ \\
\hline
\end{tabular}

and positive expression of PD-L1 (Figures 1 and 2). We found that $85.7 \%$ of TNBC specimens and $100 \%$ of ER-/ Her2+ subtypes were associated with TIL infiltration and positivity of PD-L1.

Regarding the survival data, patients with high TIL counts showed worse OS and PFS $(P=0.0238$ [HR 4.7, 95\% CI 1.22-18.11] and $P=0.0262$ [HR 3.1, 95\% CI 1.148.59] respectively) (Figures 3 and 4; Table 3 ).

Of note, there was no correlation between PD-L1 positivity (on tumor or immune cells) and survival of patients (OS and PFS) (Table 3).

Discussion

In breast cancer, the reported frequency of PD-L1

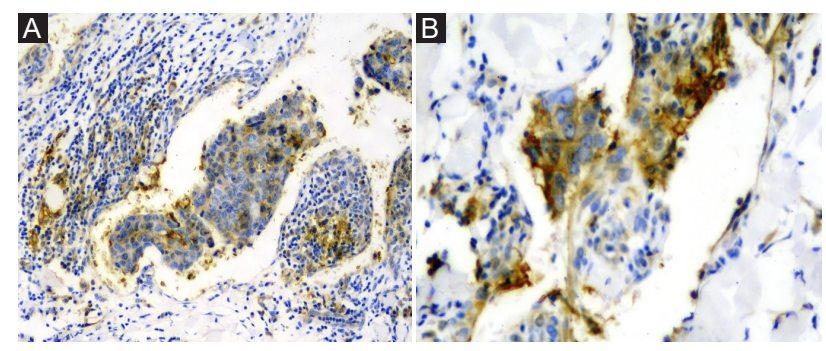

Figure 1. (A) Case of Invasive duct carcinoma grade 3 ER-/Her2 + showing moderate TIL PDL-1 expression (5-50\%) and positive PDL-1 expression in tumor cells $(>1 \%)$. (PDL1 $\times 200)$. (B) Same case showing strong positive membranous expression of PDL-1 in tumor cells surrounded by TIL (PDL-1 $\times 400)$.

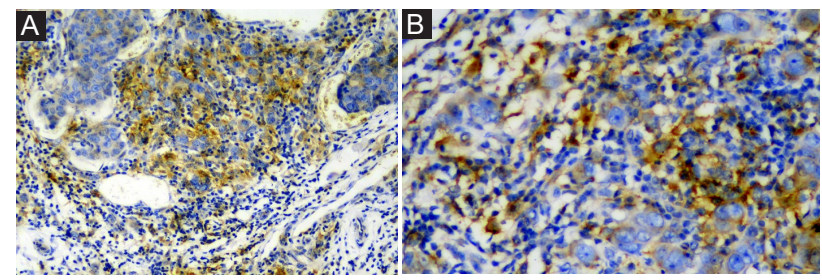

Figure 2. (A) A case of triple negative breast carcinoma grade 3 showing moderate TIL PDL-1 expression (5-50\%) and positive PDL-1 expression in tumor cells $(>1 \%)$. (PDL1 $\times 200)$. (B) Same case showing strong PDL-1 expression in $50 \%$ of TIL (PDL-1 $\times 400)$.

expression by tumor cells varies between studies (11-15). In a study that analyzed tissue from 44 patients with breast cancer, PD-L1 was expressed in 34\% of the tumors using the mouse monoclonal MIH1 clone (13). In a subsequent study conducted by the same group and using an expanded version of the cohort $(n=69)$, PD-L1 expression was expressed in $29 \%$ of breast carcinomas (14). In another report, PD-L1 was expressed in $23.4 \%$ of 650 breast cancer patients (11). A study analyzing tumors from 192 patients observed PD-L1 expression in 56\% of breast cancers (16). Another study found that PD-L1 was expressed in more than $1 \%$ of tumor cells in only $1.7 \%$ of total 3916 breast tumors by IHC analysis (12). A study analyzing tissue from 636 patients using in situ mRNA hybridization revealed that PD-L1 was expressed in $58 \%$ of the breast cancer specimens in TMAs (17). Another study using mRNA expression and DNA microarrays found that PDL1 gene expression was upregulated in $20 \%$ of all samples and $38 \%$ of basal tumors (15). All these differences may be explained by the absence of reliable antibodies, validated

Table 2. Correlation between TIL and PD-L1 on tumor cells and TILs

\begin{tabular}{|c|c|c|c|c|c|c|c|}
\hline \multirow{2}{*}{ TIL } & \multicolumn{3}{|c|}{ PD-L1 on TILs, No (\%) } & \multirow{2}{*}{$P$ value } & \multicolumn{2}{|c|}{ PD-L1 on tumor cells, No (\%) } & \multirow{2}{*}{$P$ value } \\
\hline & Focal $(n=29)$ & Low $(n=12)$ & High $(n=9)$ & & Negative $(n=37)$ & Positive (n=13) & \\
\hline Focal & $26(100)$ & $0(0)$ & $0(0)$ & \multirow{3}{*}{$<0.0001$} & $23(88.4)$ & $3(11.6)$ & \multirow{3}{*}{0.0011} \\
\hline Low & $3(25)$ & $9(75)$ & $0(0)$ & & $10(83.3)$ & $2(16.6)$ & \\
\hline High & $0(0)$ & $3(25)$ & $9(75)$ & & $4(33.3)$ & 8 (66.6) & \\
\hline
\end{tabular}

PD-L1, programmed death ligand 1 ; TIL, tumor-infiltrating lymphocytes. 


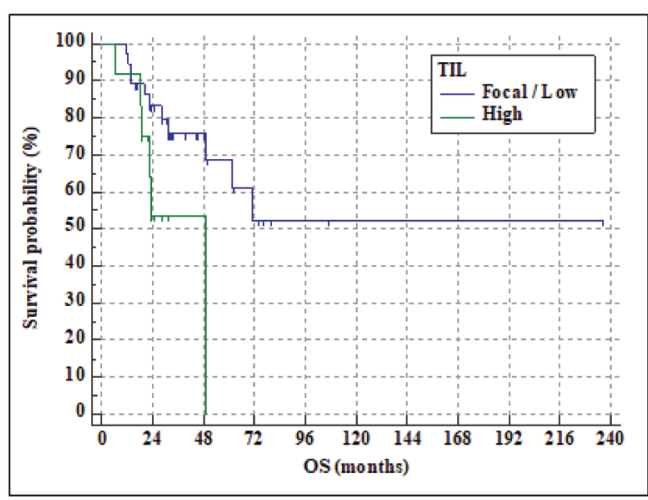

Figure 3. Kaplan Meier curve showing correlation between TILs $(<50 \%$ versus $>50 \%$ ) and OS.

assays, and interpretative uncertainties (e.g., cut off for positivity).

Blockade of the PD-L1/PD-1 pathway is a new promising therapeutic approach in oncology. We investigated the associations between TIL count/PD-L1 expression in invasive breast cancer and a number of clinicopathologic characteristics, including prognosis.

Our data reveal that PD-L1 expression was significantly associated with elevated TILs, and point to the critical role of local immunity in limiting tumor progression. This association is supported by other studies that observed the same results using in-vitro methods $(18,19)$ and with previous studies suggesting that the PD-L1/PD-1 pathway plays a major role in the induction and maintenance of immunological exhaustion in the breast tumor microenvironment $(20,21)$.

In our study, high TIL counts and PD-L1 positivity (on both TILs and tumor cells) were most significantly associated with ER-/Her2+ and TNBC. This result is supported by the results of previous studies and metaanalyses that stated that PD-L1 was most significantly associated with the triple-negative high grade tumor subtype, estrogen receptor (ER) negativity and Her2 positivity $(7,11,22-24)$.

We observed a lower level of TILs and PD-L1 expression in ductal carcinomas compared with lobular carcinomas, regardless of other pathological variables. This was in lieu with a recent study by Doğukan et al (25), while it was in contrast with a previous study that found that TILs and PD-L1 were more expressed in ductal carcinomas than lobular breast cancers (18). We have no justification for this predilection.

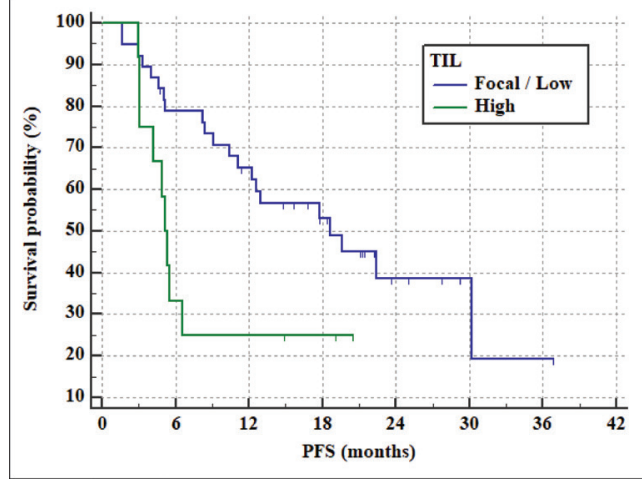

Figure 4. Kaplan Meier curve showing correlation between TILs $(<50 \%$ versus $>50 \%$ ) and PFS.

As regards the survival correlation, we found a trend towards better OS and PFS for patients with focal TIL density $(<5 \%$ infiltration) in the whole tumor population ( $P=0.0585$ and $P=0.0533$ respectively) compared to tumors with 5-50\% and tumors with $>50 \%$ TIL infiltration. The lack of significance may be due to the small number of patients included in our study. This trend became statistically significant when we grouped the patients into two subgroups (instead of three subgroups), less than and greater than $50 \%$ infiltration $(P=0.238$ and $P=0.0262$ respectively). This was different from what was stated in a recent systematic review and meta-analysis that showed that in TNBC, patients with high TIL had better OS and DFS than patients with low TIL. Studies in this metaanalysis had different definitions for 'high' TIL infiltration, yet most of the studies defined 'high' as $>50-60 \%$ TIL infiltration. This study even showed that with each $10 \%$ increase in percentage of TILs, patients with TNBC had an increased pathological complete response in patients receiving neoadjuvant treatment (26).

Regarding the relation between TIL infiltration and intrinsic subtypes, and their association with the patients' survival, the only significance we found was improved PFS for patients with focal $(<5 \%)$ TIL infiltration, compared to higher counts of infiltration $(>5 \%)$, only in patients with ER+/Her2- patients. This improvement was also found regarding the OS in the same population group but was not statistically significant.

This was in agreement with a recently published study by Kurozumi et al, that stated that TIL expression correlated with ER status and tumor proliferation. In this study, high TIL expression was a poor prognostic

Table 3. The effect of PD-L1 expression and TIL status on OS and progression free survival

\begin{tabular}{|c|c|c|c|c|c|c|c|c|c|c|}
\hline \multirow{2}{*}{ Survival } & \multicolumn{2}{|c|}{ TIL } & \multirow{2}{*}{$P$ value } & \multicolumn{3}{|c|}{ PD-L1 on immune cells } & \multirow{2}{*}{$P$ value } & \multicolumn{2}{|c|}{ PD-L1 on tumor cells } & \multirow{2}{*}{$P$ value } \\
\hline & $<50 \%$ & $>50 \%$ & & Focal & Low & High & & Negative & Positive & \\
\hline Median OS (months) & Not reached & 48.9 & 0.0238 & Not reached & Not reached & 48.9 & 0.308 & 70.8 & 61.5 & 0.8871 \\
\hline Median PFS (months) & 18.6 & 5.1 & 0.0262 & 19.6 & 10.4 & 5.5 & 0.3298 & 18.6 & 5.5 & 0.1298 \\
\hline
\end{tabular}

PD-L1=programmed death ligand 1; TIL=tumor-infiltrating lymphocytes; OS= overall survival; PFS= progression free survival. 
marker in ER-positive patients but was a good prognostic marker in ER-negative patients. In this study, TIL grades were categorized into low $(<10 \%)$, intermediate $(\geq 10$ and $\leq 40 \%)$ and high (>40\%) groups. Therefore, Kurozumi et al concluded that the biological association between TILs and primary breast tumors may differ between ER-positive and ER-negative breast cancer (27).

These results were in contradiction to what Sobral et al found, where in their study, TILs infiltration showed no association with breast cancer specific survival (BCSS) in ER+/Her2- and Her2+ tumors, while high TIL status was significantly associated with better BCSS in only TNBC tumors (7). As regards the PD-L1 expression on tumor cells and TILs, we did not find any statistically significant correlation between its positivity, and the survival of the patients (OS and PFS). This non-significance may be also attributed to the low number of patients in our study.

These non-significant results were also found in a previous trial, that found that $\mathrm{PD}-\mathrm{L} 1+$ tumor co-expression showed no correlation with survival of the patients (7).

A meta-analysis by Zhang et al, found that PD-L1 overexpression was associated with shorter OS in patients with breast cancer. This was attributed to the finding that in this meta-analysis, higher PD-L1 expression was linked to patients with poor clinico-pathologic criteria; as positive lymph nodes, higher histologic grades and ER negativity. The same results were also confirmed by another meta-analysis, that also demonstrated that PD-L1 over-expression was significantly associated with positive lymph node metastasis, poor nuclear grades, and ER negativity in breast cancer patients (28).

In a large previously mentioned meta-analysis by Huang et al, PD-L1+ tumor cells were significantly associated with shorter disease-free survival and OS (23).

A recent Chinese study found that $\mathrm{PD}-\mathrm{L} 1$ positive tumor cells (PD-L1+ tumor) in TNBC patients had significantly longer DFS and OS values than those of PD-L1 negative tumor cells ( $P=0.046 ; P=0.019$ respectively) (29).

In another recent meta-analysis, positive PD-L1 on TILs was associated with better survival of all patients in the pooled analysis, especially TNBC basal subtype, while positive over-expression of PD-L1 on tumor cells had a negative impact on survival of all patients especially also the TNBC basal like subtype (30).

\section{Conclusion}

There is a major controversy about the prognostic value of TIL and PD-L1 in breast cancer. TNBC and Her2 enriched are the most common types associated with TIL infiltration and PD-L1 positivity (on tumor cells and TILs), and this renders them more immunogenic than other subtypes. This is especially important in TNBC because of the lack of any targeted therapeutic solution other than chemotherapy. Further studies are warranted to determine the predictive value of PD-L1 positivity and TIL infiltration regarding the response to immunotherapy.
Limitations of the study

Our study included 50 patients, which could be of relatively limited sample size. Although the results reached a statistically significant correlation between PDL1 expression and TNBC, yet it did not correlate with patients' PFS and OS, which could be attributed to the limited number.

Authors' contribution

MMD: Collection and analysis of data, extraction of data from patient records, design research methodology AYR, DAS, AEE, DEMS: idea and study plan, supervision, revision of data analysis and manuscript revision MMS: revision of pathology reports and immunostains, scoring, counting and photographing of TIL, PDL1 immunohistochemical expression, supervision, revision and submission of the manuscript. All authors have read and approved the content of the manuscript and confirmed the accuracy or integrity of any part of the work.

Conflicts of interest

The authors declare that they have no competing interests.

\section{Ethical issues}

The research followed the tenets of the Declaration of Helsinki. The institutional ethical committee at Ain Shams University, Faculty of Medicine approved all study protocols (IRB approval number; MD 271/2018). The study was based on data collection and immunohistochemical analysis of positively charged slides prepared from paraffin blocks. Accordingly, informed consent was obtained at their admission time. This study was extracted from M.D thesis of Mohamed Mostafa Darwish at this university. Additionally, ethical issues (including plagiarism, data fabrication, double publication) were completely observed by the authors.

Funding/Support

The authors declare there are no funding sources.

\section{References}

1. Bray F, Ferlay J, Soerjomataram I. Global Cancer Statistics 2018: GLOBOCAN Estimates of Incidence and Mortality Worldwide for 36 Cancers in 185 Countries. CA Cancer J Clin. 2018;68:394-424. doi: 10.3322/caac.21492.

2. Zerdes I, Matikas A, Bergh J, Rassidakis GZ, Foukakis T. Genetic, transcriptional and post-translational regulation of the programmed death protein ligand 1 in cancer: biology and clinical correlations. Oncogene. 2018;37:4639-61. doi: 10.1038/s41388-018-0303-3.

3. Li J, Gu J. Efficacy and safety of PD-1 inhibitors for treating advanced melanoma: A systematic review and meta-analysis. Immunotherapy. 2018;10:1293-302. doi: 10.2217/imt-20180116.

4. Zhou Y, Chen C, Zhang X, Fu S, Xue C, Ma Y, et al. Immunecheckpoint inhibitor plus chemotherapy versus conventional chemotherapy for first-line treatment in advanced non-small cell lung carcinoma: A systematic review and meta-analysis. J Immunother Cancer. 2018;6:1-11. doi: 10.1186/s40425-0180477-9.

5. Wang $\mathrm{H}$, Zhang $\mathrm{C}$, Zhang J, Kong L, Zhu H, Yu J. Effectiveness and safety of PD-1/PD-L1 inhibitors in the treatment of solid tumors: A systematic review and meta-analysis. Oncotarget. 2017;8:59901-14. doi: 10.18632/oncotarget.18316.

6. Schmid P, Adams S, Rugo H, Schneeweiss A, Barrios C. Atezolizumab and Nab-paclitaxel in advanced triple-negative breast cancer IMpassion150. N Engl J Med. 2018;79:232. doi: 10.1055/a-0832-5840. 
7. Sobral-Leite M, Van de Vijver K, Michaut M, van der Linden R, Hooijer GK, Horlings H. M. Assessment of PD-L1 expression across breast cancer molecular subtypes, in relation to mutation rate, BRCA1-like status, tumor-infiltrating immune cells and survival. Oncoimmunology. 2018;7:1-15. doi: 10.1080/2162402X.2018.1509820.

8. Schmid P, Adams S, Rugo HS, Schneeweiss A, Barrios $\mathrm{CH}$, Iwata $\mathrm{H}$ et al. Atezolizumab and Nab-Paclitaxel in Advanced Triple-Negative Breast Cancer. N Engl J Med. 2018;379:21082121. doi: 10.1056/nejmoa1809615.

9. Emens LA, Cruz C, Eder JP, Braiteh F, Chung C, Tolaney SM, et al. Long-term Clinical Outcomes and Biomarker Analyses of Atezolizumab Therapy for Patients with Metastatic TripleNegative Breast Cancer: A Phase 1 Study. JAMA Oncol. 2019;5:74-82. doi: 10.1001/jamaoncol.2018.4224

10. Salgado R, Denkert C, Demaria S, Sirtaine N, Klauschen F, Pruneri G, et al. The evaluation of tumor-infiltrating lymphocytes (TILS) in breast cancer: Recommendations by an International TILS Working Group 2014. Ann Oncol. 2015;26:259-71. doi: 10.1093/annonc/mdu450.

11. Muenst S, Schaerli AR, Gao F, Däster S, Trella E, Droeser RA, et al. Expression of programmed death ligand 1 (PD-L1) is associated with poor prognosis in human breast cancer. Breast Cancer Res Treat. 2014;146:15-24. doi: 10.1007/s10549-0142988-5.

12. Ali HR, Glont SE, Blows FM, Provenzano E, Dawson SJ, Liu B, Hiller L, et al. PD-L1 protein expression in breast cancer is rare, enriched in basal-like tumours and associated with infiltrating lymphocytes. Ann Oncol. 2015;26:1488-93. doi: 10.1093/annonc/mdv192.

13. Ghebeh H, Mohammed S, Al-Omair A, Qattant A, Lehe C, AlQudaihi G, et al. The B7-H1 (PD-L1) T lymphocyte-inhibitory molecule is expressed in breast cancer patients with infiltrating ductal carcinoma: correlation with important high-risk prognostic factors. Neoplasia. 2006;8:190-8. doi: 10.1593/ neo.05733.

14. Ghebeh H, Tulbah A, Mohammed S, ElKum N, Amer SM, AITweigeri $\mathrm{T}$, et al. Expression of B7-H1 in breast cancer patients is strongly associated with high proliferative Ki-67-expressing tumor cells. Int J Cancer. 2007;121:751-8. doi: 10.1002/ ijc. 22703 .

15. Sabatier R, Finetti P, Mamessier E, Adelaide J, Chaffanet M, Ali $H R$, et al. Prognostic and predictive value of PDL1 expression in breast cancer. Oncotarget. 2015;6:5449-64. doi: 10.18632/ oncotarget.3216.

16. Baptista MZ, Sarian LO, Derchain SF, Pinto GA, Vassallo J. Prognostic significance of PD-L1 and PD-L2 in breast cancer. Hum Pathol. 2016;47:78-84. doi: 10.1016/j. humpath.2015.09.006

17. Schalper KA, Velcheti V, Carvajal D, Wimberly $H$, Brown J. Pusztai L, et al. In situ tumor PD-L1 mRNA expression is associated with increased TILs and better outcome in breast carcinomas. Clin Cancer Res. 2014;20:2773-82. doi: 10.1158/1078-0432.CCR-13-2702.

18. Buisseret L, Garaud S, de Wind A, Van den Eynden G, Boisson A, Solinas C, et al. Tumor-infiltrating lymphocyte composition, organization and PD-1/PD-I1 expression are linked in breast cancer. Oncoimmunology. 2017;6:e1257452. doi: 10.1080/2162402X.2016.1257452.

19. Nolan E, Savas P, Policheni AN, Darcy PK, Vaillant F, Mintoff $\mathrm{CP}$, et al. Combined immune checkpoint blockade as a therapeutic strategy for BRCA1-mutated breast cancer. Sci Transl Med. 2017;9:1-13. doi: 10.1126/scitranslmed.aal4922.

20. Cimino-Mathews A, Thompson E, Taube JM, Ye X, Lu Y, Meeker A, et al. PD-L1 ( B7-H1) expression and the immune tumor microenvironment in primary and metastatic. Hum Pathol. 2016;47:52-63. doi: 10.1016/j.humpath.2015.09.003.

21. Emens LA, Kok M, Ojalvo LS. Targeting the programmed cell death-1 pathway in breast and ovarian cancer. Curr Opin Obstet Gynecol. 2016;28:142-147. doi: 10.1097/ GCO.0000000000000257

22. Wang ZQ, Milne K, Derocher H, Webb JR, Nelson BH, Watson PH. PD-L1 and intratumoral immune response in breast cancer. Oncotarget. 2017;8:51641-51. doi: 10.18632/ oncotarget.18305.

23. Huang W, Ran R, Shao B, Li H. Prognostic and clinicopathological value of PD-L1 expression in primary breast cancer: a meta-analysis. Breast Cancer Res Treat. 2019;178:17-33. doi: 10.1007/s10549-019-05371-0.

24. Kitano A, Ono M, Yoshida M, Noguchi E, Shimomura A, Shimoi $\mathrm{T}$, et al. Tumour-infiltrating lymphocytes are correlated with higher expression levels of PD-1 and PD-L1 in early breast cancer. ESMO Open. 2017;2:e000150. doi: 10.1136/ esmoopen-2016-000150.

25. Doğukan R, Uçak R, Doğukan FM, Tanık C, Çitgez B. Correlation between the Expression of PD-L1 and Clinicopathological Parameters in Triple Negative Breast Cancer Patients. Eur J Breast Heal. 2019;15:235-41. doi: 10.5152/ejbh.2019.4912.

26. Gao G, Wang Z, Qu X, Zhang Z. Prognostic value of tumorinfiltrating lymphocytes in patients with triple-negative breast cancer: A systematic review and meta-analysis. BMC Cancer. 2020;20:179. doi: 10.1186/s12885-020-6668-z.

27. Kurozumi S, Matsumoto H, Kurosumi M, Inoue K, Fujii, T., Horiguchi J, et al. Prognostic significance of tumour-infiltrating lymphocytes for oestrogen receptor-negative breast cancer without lymph node metastasis. Oncol Lett. 2019;17:2647-56. doi: 10.3892/ol.2019.9938.

28. Guo Y, Yu P, Liu Z, Maimaiti Y, Wang S, Yin X, et al. Prognostic and clinicopathological value of programmed death ligand-1 in breast cancer: A meta-analysis. PLoS One. 2016;11:e0156323. doi: 10.1371/journal.pone.0156323.

29. AiErken N, Shi Hj, Zhou Y, Shao N, Zhang J, Shi Y, et al. High PD-L1 Expression Is Closely Associated With Tumor-Infiltrating Lymphocytes and Leads to Good Clinical Outcomes in Chinese Triple Negative Breast Cancer Patients. Int J Biol Sci 2017;13:1172-9. doi: 10.7150/ijbs.20868.

30. Matikas A, Zerdes I, Lövrot J, Richard F, Sotiriou C, Bergh J, et al. Prognostic implications of PD-L1 expression in breast cancer: Systematic review and meta-analysis of immunohistochemistry and pooled analysis of transcriptomic data. Clin Cancer Res. 2019;25:5717-26. doi: 10.1158/1078-0432.CCR-19-1131. 